\title{
A new co-therapeutic strategy for RA?
}

B cells have a crucial role in driving autoimmunity in rheumatoid arthritis (RA). Indeed, B-cell-depletion therapy involving the anti-CD20 antibody rituximab can be an effective therapeutic strategy for patients with RA. The improvement in clinical symptoms of these patients, however, is temporary and B cell populations eventually return, along with RA symptoms. Now, Pigott and Mandik-Nayak have published in Arthritis $\&$ Rheumatism that the addition of an inhibitor of indoleamine-2,3-dioxygenase (IDO) - an enzyme that drives the initial stages of the B-cell-mediated autoimmune response-to B-cell-depletion therapy could promote long-term clinical improvement in patients with RA.

The authors used immunoglobulin transgenic and arthritic transgenic mice to test the efficacy of the IDO inhibitor 1-methyltryptophan (1MT) in vivo. 1MT did not affect initial activation or survival of autoreactive B cells but did prevent their ability to differentiate into autoantibody-secreting cells. In arthritic mice rituximab was effective in depleting the number of B cells and attenuating RA symptoms, but only for a limited period. Administration of $1 \mathrm{MT}$ prior to B-cell repopulation prevented the production of autoantibodies and inflammatory cytokines and flare in arthritis symptoms.

These findings indicate that IDO inhibitors and rituximab could be an effective co-therapeutic strategy for patients with RA by both eliminating pathogenic B cells and preventing new ones from being generated. Moreover, $1 \mathrm{MT}$ should not affect memory responses to pathogens acquired through vaccination. Indeed, $1 \mathrm{MT}$ is currently in early stage clinical trials as an anti-cancer therapeutic.

\section{Katherine Smith}

Original article Pigott, E. \& Mandik-Nayak, L. Addition of an indoleamine-2,3,-dioxygenase inhibitor to B cell depletion therapy blocks autoreactive $B$ cell activation and recurrence of arthritis. Arthritis Rheum. doi 10.1002/ art.34406. 